\title{
Vancomycin use in a Brazilian teaching hospital: comparison with the Hospital Infection Control Practices Advisory Committee Guidelines (HICPAC)
}

\author{
Daniela Oliveira de Melo ${ }^{1}$ and Eliane Ribeiro ${ }^{2}$ \\ ${ }^{1}$ University of São Paulo - Pharmaceutical Sciences School; ${ }^{2}$ University of São Paulo - Pharmaceutical Sciences School and University \\ Hospital; São Paulo, SP, Brazil
}

\begin{abstract}
This study describes vancomycin prescribing patterns in an average complexity hospital and compare the guidelines proposed by the Hospital Infection Control Practices Advisory Committee (HICPAC). The study was conducted in a 256-bed secondary-care hospital. Data were collected of all patients given vancomycin from March 2003 to February 2004, using a standardized chart-extraction form designed. Appropriate and inappropriate use was reviewed according to the Hospital Infection Control Practices Advisory Committee (HICPAC) guidelines on prudent vancomycin use. Out of 118 prescriptions, $95(80.5 \%)$ were considered appropriate. Out of these 95 orders, $77(81.1 \%)$ were administered for empiric treatment of suspected Gram-positive infections, $17(17.9 \%)$ were administered for treatment of proven Gram-positive infections (76.5\% identified as Staphyloccocus aureus-like agents) and 1 (1.0\%) for beta-lactam allergy. The majority of the patients $(\mathbf{9 6 . 6 \% )}$ ) had recently used an antimicrobial medication (3 months). The mean pre-treatment hospitalization period was $11 \pm 10$ days. Out of the 118 treatments, 67 (56.8\%) were for nosocomial infections. The more frequent indications for vancomycin use were pneumonia (48.3\%) and primary sepsis (18.6\%), accounting for more than $66 \%$ of all treatments. No restriction policy was suggested because vancomycin use was considered adequate in the majority of the treatment cases. The broad empiric use of this antimicrobial was greater than expected in the institution and its use should be revised.

Key-Words: Vancomycin, infection, guidelines, antimicrobial use.
\end{abstract}

The development of resistance to multiple drugs in Grampositive pathogens is a serious clinical problem. Inadequate empiric therapy enhances the potential risk of developing resistance by $50 \%$ to $60 \%[1,2]$.

The increasing incidence of vancomycin enterococci (VRE) and the potential transfer of genetic factors responsible for vancomycin resistance to different Gram-positive microorganisms, including Staphylococcus aureus [3-7] is an international concern. Infections caused by strains of Staphylococcus aureus with reduced susceptibility to vancomycin (minimum inhibitory concentration $=8 \mu \mathrm{g} / \mathrm{mL}$ ) have been described. In Japan and in the United States. All cases occurred following a prolonged course of vancomycin therapy [8-10]. In the United States cases of vancomycin resistant Staphylococcus aureus (VRSA) clinical infections were reported, in 2002 [7,11-13]. S. aureus intermediately resistant to Vancomycin has been described in Brazil [14-16].

Enterococcus sp. is the second most commonly isolated nosocomial pathogen. It is responsible for $8 \%$ of hospital acquired bloodstream infections $[17,18]$. The prevalence of vancomycin-resistant enterococcus (VRE) infections increased 20-fold over the past 10 years [19,20], especially in high-risk patient populations. Between 1989 and 1993, the proportion of enterococcal isolates resistant to vancomycin among in-patients increased from $0.3 \%$ to $7.9 \%$ and from $0.4 \%$ to $13.6 \%$ in intensive-care unit patients [21].

Received on 15 February 2009; revised 16 May 2009.

Address for correspondence: Dr. Daniela Oliveira de Melo. Rua Anibal Pedro Godinho, 286, Jd Esther Yolanda. Zip code: 05374-200. São Paulo - SP, Brazil.

The Brazilian Journal of Infectious Diseases 2009;13(3):161-164. (C) 2009 by The Brazilian Journal of Infectious Diseases and Contexto Publishing. All rights reserved.
Over the last decade, vancomycin-resistant enterococci (VRE) have become a common cause of nosocomial infections in Western Europe and in the United States [4,20,22-26]. The occurrence of VRE in National Nosocomial Infections Surveillance (NNIS) hospitals has been associated with larger size hospitals (i.e., a hospital with at least 200 beds) and university affiliation [5,27]. The NNIS reported a vancomycin resistance rate of $26.3 \%$ for enterococci isolated from patients with nosocomial infections in intensive care units (ICUs) in 2000 [28,29]. In Brazil, the first case was reported in São Paulo [30]. Other cases occurred in different hospitals and, by 1998, VRE had spread throughout the country [31]. Disease severity controlled studies found that vancomycin resistance was predictive of increased mortality [32-34]. Comparing patients with vancomycin-susceptible isolates, patients with VRE bacteremia exhibit significantly increased mortality rates (17.2\% vs. $36.0 \%)[18,21]$.

In 1995, the Centers for Disease Control and Prevention (CDC) and the Hospital Infection Control Practices Advisory Committee (HICPAC) issued guidelines to prevent the spread of vancomycin resistance [5]. Several studies demonstrated the benefit of stool scrutiny in decreasing the rate of VRE infections in healthcare facilities [4,22-25,32]. The objective of this study was to evaluate intravenous vancomycin use at a secondary-care teaching hospital and to estimate the proportion of intravenous vancomycin prescribed for an indication meeting the HICPAC guidelines, using actual information available in chart-review.

\section{Material and Methods}

The University Hospital provides services to the university community and local population and has a 10-bed intensive care unit and a 10-bed semi intensive care unit. It is 
a secondary-care teaching hospital, with 256 beds. The Pharmacy Service is centralized and attends hospitalized patients on an individual basis. Prescriptions are reviewed in each clinic by the pharmacist in charge. Drugs are dispensed to patients, for a maximum period of 24 hours (doses for oral or enteral administration are dispensed ready for use and doses for potential administration are dispensed without manipulation, with the identification of product and patient, dilution calculation and administration precautions).

\section{Study Population}

The Study Population included Patients who received intravenous vancomycin between March 2003 and February 2004, as in-patients of a 256-bed, secondary-care teaching hospital. This hospital provides services to the university community and local population and has a 10-bed intensive care unit and a 10-bed semi intensive care unit.

\section{Data Collection and Analysis}

All new vancomycin prescriptions were evaluated. The order form identified new cases. A standard design chartextraction form was used for entering the data. These data included underlying diagnosis, history of drug allergy, demographic characteristics, hospital location, indications for vancomycin use, including culture and sensitivity data, antimicrobial treatment in the preceding three months, nature of infection, suspension of treatment when necessary or possible and antimicrobial replacement, renal insufficiency and dosing regimen.

\section{Appropriate Use Criteria}

Use was considered appropriate if the clinical indication met the HICPAC criteria. In cases of empiric use in patients with risk factors due to hospital epidemiology - high prevalence of methicillin-resistant Staphylococcus aureus (MRSA). Accordingly, the Hospital Infection Control Commission identified during the study period nine nosocomial infections caused by Staphylococcus aureus. Out of these, eight were MRSA.

\section{Inappropriate Use was Subdivided}

a. Use of empiric therapy without the presence of risk factors;

b. Continued empiric use for presumed infections in patients whose cultures were negative for beta-lactam-resistant Gram-positive microorganisms;

c. Treatment of infections caused by beta-lactam-sensitive Gram-positive microorganisms, without allergy history to beta-lactam antimicrobials;

d. Treatment in response to a single positive blood culture for coagulase-negative staphylococcus, if other blood cultures taken during the same time-frame are negative;

e. Systemic or local prophylaxis for infection or colonization of indwelling central or peripheral intravascular catheters.
Secondary Conformity Analysis Classification of Inappropriate Use

Vancomycin use was studied after the culture results became available. If cultures revealed methicillin-sensitive staphylococci, or another relevant pathogen, maintenance of vancomycin use was considered inappropriate.

\section{Renal Function Monitoring and Dosing Regimen Correction}

Renal function monitoring was reviewed (serum urea or creatinine). When these tests were performed at least twice a week, monitoring was considered adequate. The dosing regimen, including adjustment for renal insufficiency, was evaluated using the normogram [35].

\section{Results}

Vancomycin was prescribed 118 times during the year audited (March 2003 to February 2004). The average age was $49 \pm 29$ years. The mean pre-treatment hospitalization period was $11 \pm 10$ days. The most frequent indications for vancomycin use were pneumonia (48.3\%), septicemia (18.6\%), peritonitis (7.6\%), catheter local infection (5.1\%), surgical wound infection (4.2\%), and meningitis (2.5\%). The medical specialties that prescribed the drug most were: intensive care unit (37.3\%), semi-intensive care unit (16.9\%), medical clinic (13.6\%), surgical clinic (11.0\%) and pediatric intensive care unit (10.2\%).

Out of the 118 treatments, 67 (56.8\%) were nosocomial infections, 11 (9.3\%) were healthcare-related infections and 40 (33.9\%) community infections. The majority of the patients (96.6\%) had used antimicrobials recently (3 months).

Vancomycin use was considered consistent with the HICPAC guidelines in 95 (80.5\%) patients. Out of these, 77 (81.1\%) for empiric treatment of suspected Gram-positive infections, 17 (17.9\%) for treatment of proven Gram-positive infections (76.5\% identified Staphyloccocus aureus-like agents) and 1 (1.0\%) for beta-lactam allergy (Table 1$)$.

Out of the 95 treatments considered appropriate, 5 (5.3\%) had cultures that justified the antimicrobial discontinuation but only 3 (3.2\%) actually had the antimicrobial suspended. Among the 23 (19.5\%) treatments considered inappropriate, 15 (65.2\%) were community infections and 8 (34.8\%) were treatments for agents that did not warrant that treatment.

Table 1. Results of vancomycin audit.

\begin{tabular}{lr}
\hline Use & N (\%) \\
\hline Appropriate & $95(80.5 \%)$ \\
Empiric therapy with risk factors & $77(81.1 \%)$ \\
Treatment of proven Gram-positive & $17(17.9 \%)$ \\
infections & $1(1.0 \%)$ \\
Beta-lactam allergy & $23(19.5 \%)$ \\
Inappropriate & $15(65.2 \%)$ \\
$\quad \begin{array}{l}\text { Empiric therapy without risk factors; } \\
\text { Continued empiric use without further } \\
\text { evidence of Gram-positive infection }\end{array}$ & $8(34.8 \%)$ \\
\hline
\end{tabular}


Among the 23 cases of inappropriate treatment, 7 (26.1\%) began in a medical clinic, 6 (26.1\%) in intensive care units, 4 (17.4\%) in pediatric intensive care units, 3 (13.0\%) in a surgical clinic, 2 (8.7\%) in semi-intensive care units and 1 (4.3\%) in a nursery.

The average treatment duration was $12 \pm 10$ days. The major dosing regimen employed was 1 gram every 12 hours and 500 milligrams every 6 hours, in 52 (44.1\%) and 7 (5.9\%) treatments, respectively. The other dosing regimens were specifically adjusted for renal insufficiency. Five patients, 60 years old or over, used a dosing regimen of 1 gram every 24 hours, despite the absence of renal insufficiency. This is justified by the slow metabolism [35]. Out of 23 children, 2 (8.7\%) received a dose of the antimicrobial that was greater than necessary.

When the report on renal insufficiency was assessed, before or during the treatment with the antimicrobial, fortyfour patients (37.3\%) reported suffering from insufficiency before the treatment and 17 (14.4\%) after its use. The function was monitored in 123 (93.4\%) patients and the dose wasn't corrected for $2(5.9 \%)$ patients who presented renal insufficiency (Table 2). Among the patients not monitored, 5 (35.7\%) were children.

Table 2. Adjustment of posology, according to renal function.

\begin{tabular}{lcl}
\hline Adjustment & N (\%) \\
\hline Not required & $57(48.3 \%)$ \\
Required & $61(51.7 \%)$ \\
$\quad$ Correctly adjusted & $57(93.4 \%)$ \\
Incorrectly adjusted & $2(3.3 \%)$ \\
No correction performed & $2(3.3 \%)$ \\
\hline
\end{tabular}

\section{Discussion}

In March 2003 another study on the use of vancomycin in a Brazilian tertiary hospital was initiated by one of the authors of this paper. It is of notice that in both hospitals, MRSA prevalence is high, which justifies the empiric use of vancomycin in the treatment of hospital infections. MRSA prevalence in $\mathrm{HU}$ is $41 \%$ and 92 (78\%) patients received empiric treatment for suspected Gram-positive infections, which is consistent with the results of the study in the other Brazilian hospital (78\% of the empiric treatment with vancomycin) [36].

The empiric use in other studies $[8,27,37]$ varied between $33 \%$ and $71 \%$, among all patients that received vancomycin, Success rates in the management of Gram-positive infections (post surgical patients, ventilator-associated pneumonia, peritonitis, bacteremia and meningitis) were enhanced by early therapy with active agents against the organisms later identified by the appropriate culture. Inadequate therapy is closely correlated with adverse patient outcomes, including increased rates of hospital mortality [35,38,39].

Only one study confirmed an MRSA proportion considered high, more than 20\% MRSA [40]. The empiric use was responsible for $81.1 \%$ of the prescriptions considered adequate, because of hospital epidemiology (Table 1). In a Brazilian tertiary hospital, the empiric treatment was responsible for $81.1 \%$ of appropriate vancomycin use (because of hospital epidemiology) and 65.2\% was inappropriate [36]. In other studies, the empiric treatment with vancomycin was considered the second largest category of inappropriate use (26\%), and in others two studies it was considered the first, with $67 \%$ of inappropriate use $[8,27,37]$.

For instance, the HICPAC guidelines state that vancomycin is an appropriate treatment for infections caused by Grampositive microorganisms in patients with serious allergies to beta-lactam antibiotics; we considered as "appropriate", the only case of allergy that was reported in a patient's chart although the severity of the allergy was not specified.

According to Morgan et al. (1997), the average treatment duration was 4.7 days, influenced by the prophylactic use [27]. The prophylactic use is very common in the United Sates, but less frequent in Brazil. Failure to discontinue the documented inadequate treatments indicates that greater attention to a review of the medical conduct is required. No correction of dosing regimen occurs when there is renal insufficiency concern, mainly because the hospital attends an older population.

Surveillance of the posological scheme was inappropriate. Vancomycin misuse may be associated with clinical failure, toxicity or super-infections, such as Gram-negative bacteremia in children [41]. The higher dose regimen in children demonstrates failures in this antimicrobial prescription and dispensation system, considering an ordinarily corrected order by the clinic pharmacist. Vancomycin use was strongly associated with Gram-negative bacteremia in children [41]. An explanation was that severe infectious diseases, predominantly caused by Gram-negative bacteria, induce the use of central venous catheters, which in turn induces the use of vancomycin. However, the association between vancomycin and Gram-negative bacteremia is still regarded as strong when the study was restricted to children without central venous catheter [41].

Studies suggest that, even in the absence of restriction policies, vancomycin use is not more than $40 \%$ of the guidelines recommendations $[36,38,39,42]$. Other studies have shown that antibiotic control measures can reduce total vancomycin use [18,39]. Additionally, the implementation of pharmacy policies designed to reduce the number of initial orders of vancomycin were associated with a reduction of the inappropriate use of this antimicrobial and rate of VRE infections in healthcare facilities [1,8,20,22-25,35]. Hospital pharmacies represent an even more important control point for this drug [4].

In the institution analyzed, vancomycin use was considered cautious. We believe that other antimicrobials require more attention and no restriction policy was suggested. But, due to the large empiric use of these antimicrobials there is a consensus that vancomycin use should be monitored.

\section{Acknowledgements}

Financial support: University Hospital; University of São Paulo. 


\section{References}

1. Edmond M.B., Wallace S.E., McClish D.K. et al. Nosocomial bloodstream infections in United States hospitals: a three-year analysis. Clin Infect Dis 1999;29:239-44.

2. Richards M.J., Edwards J.R., Culver D.H., Gaynes R.P. Nosocomial infections in combined medical-surgical intensive care units in the United States. Infect Control Hosp Epidemiol 2000; 21:510-15.

3. Solomkin J.S., Bjornson H.S., Cainzos M. et al. A consensus statement on empiric therapy for suspected Gram-positive infections. The Am J of Surg 2004;187:134-145.

4. Cieslak P.R., Strausbaugh L.J., Fleming D.W., Ling J.M. Vancomycin in Oregon: who's using it and why. Infect Control Hosp Epidemiol 1999;20(8):557-560.

5. Centers of Disease Control and Prevention. Recommendations for preventing the spread of vancomycin resistance Recommendations Infection Control Practices Advisory Committee (HICPAC). MMWR 1995; 44(RR12): 1-13.

6. Noble W.C., Virani Z., Cree R. Co-transfer of vancomycin and other resistance genes from Enterococcus faecalis NCTC12201 to Staphylococcus aureus. Microbiol Lett 1992;72:195-8.

7. Smith T.L., Pearson M.L., Wilcox K.R. et al. emergence of vancomycin resistance in Staphylococcus aureus. GlycopeptideIntermediate Staphylococcus aureus working Group. N Engl J Med 1999;340:493-501.

8. Thomas R.A., Cieslak R.P., Straugsbaugh J.L., Fleming W.D. Effectiveness of pharmacy policies designed to limit inappropriate vancomycin use: A population- based assessment. Infect Control Hosp Epidemiol 2002;23:683-8.

9. Hiramatsu K., Hanaki H., Ino T. et al. Methicillin-resistant Staphylococcus aureus clinical strengh with reduced vancomycin susceptibility. J Antimicrob Chemother 1997;40:135-6.

10. Rotun S.S., McMath V., Schoonmaker D. et al. Staphylococcus aureus with reduced susceptibility to vancomycin isolated from a patient with fatal bacteremia. Emerg Infect Dis 1999;5:147-9.

11. Centers for Disease Control and Prevention. Staphylococcus aureus with reduced susceptibily to vancomycin: Illinois, 1999. MMWR 2000;48:1165-7.

12. Centers for Disease Control and Prevention. Public Health dispatch: Vancomycin-resistant Staphylococcus aureus - Pennsylvania, 2002. MMWR 2002;51(40):902-3.

13. Centers for Disease Control and Prevention. Staphylococcus aureus resistant to vancomycin - United States, 2002. MMWR 2002;51:565-7.

14. Camargo I.L., Zanella R.C., Brandileone M.C. et al. Occurrence of insertion sequences within the genomes and Tn 1546-like elements of glycopeptide-resistant enterococci isolated in Brazil, and identification of a novel element, ISEfa5. Int J Med Microbiol 2005;294(8):513-9.

15. Palazzo I.C., Araujo M.L., Darini A.L. First report of vancomycinresistant staphylococci isolated from healthy carriers in Brazil. J Clin Microbiol 2005;43(1):179-85.

16. Kluytmans-Vandenbergh M.F., Kluytmans J.A. Communityacquired methicillin-resistant Staphylococcus aureus: current perspectives. Clin Microbiol Infect 2006;12 Suppl 1:9-15.

17. Schaberg D.R., Culver D.H., Gaynes R.P. Major trends in microbial etiology of nosocomial infection. Am J Med 1991;919(suppl 3B):72S-5S.

18. Lautenbach E., Biulker W.B., Brennan P.J. Enterococcal bacterimia: risk factors for vancomycin resistance and predictors of mortality. Infect Control Hosp Epidemiol 1999;20:318-23.

19. National Nosocomial Infections Surveillance System. National Nosocomial Infections Surveillance (NNIS) System report: data summary from October 1986-April 1998, issued June 1998. Am J Infect Control 1998;26:522-33.

20. Hachem R., Graviss L., Hanna H. et al. Impact of surveillance for vancomycin-resistant enterococci on controlling a bloodstream outbreak among patients with hematologic malignancy. Infect Control Hosp Epidemiol 2004;25:391-4.

21. Centers for Disease Control and Prevention. Nosocomial enterococci resistant to vancomycin - United Sates, 19891993. MMWR 1993;42:597-9.
22. Grayson M.L., Grabsch E.A., Johnson P.D. et al. Outcome of a screening program for vancomycin-resistant enterococci in a hospital in Victoria. Med J Aust 1999;171:133-6.

23. Hendrix C.W., Hammond J.M.J., Swoboda S.M. et al. Surveillance strategies and impact of vancomycin-resistant enterococcal colonization and infection in critically ill patients. Ann Surg 2001;233:259-65.

24. Price C.S., Paule S., Noskin G.A., Peterson L.R. Active surveillance reduces the incidence of vancomycin-resistant enterococcal bacteremia. Clin Infect Dis 2003;37:921-8.

25. Montecalvo M.A., Jarvis W.R., Uman J. et al. Infection-control measures reduce transmission of vancomycin-resistant enterococci in an endemic setting. Ann Intern Med 1999;131:269-72.

26. Ostrowsky B.E., Trick W.E., Sohn A.H. et al. Control of vancomycin-resistant Enterococcus in health care facilities in a region. N Engl J Med 2001;344:1427-33.

27. Morgan S.A., Brennan J.P., Fishman O.N. Impact of a vancomycin restriction policy on use and cost of vancomycin and incidence of vancomycin-resistant Enterococcus. Ann Pharmacother 1997;31:1,970-3.

28. National Nosocomial Infections Surveillance (NNIS) System. National Nosocomial Infections Surveillance System report: data summary from January 1992-June 2001. Am J Infect Control 2001;29:404-21.

29. Christiansen K.J., Tibbett P.A., Beresfor W. et al. Eradication of a large outbreak of a single strain of van B vancomycin-resistant Enterococcus faecium at a major Australian teaching hospital. Infect Control Hosp Epidemiol 2004;25:384-90.

30. Zanella R.C., Valdetaro F., Lovgren M. et al. First confirmed case of a vancomycin-resistant Enterococcus faecium with vanA phenotype from Brazil: isolation from a meningitis case in Sao Paulo. Microb Drug Resist 1999;5(2):159-62.

31. Cereda R.F., Gales A.C., Silbert S. et al. Molecular typing and antimicrobial susceptibility of vancomycin-resistant Enterococcus faecium in Brazil. Infect Control Hosp. Epidemiol 2002;23(1):19-22.

32. Edmond M.B., Ober J.F., Dawson J.D. et al. Vancomycin-resistant enterococcal bacteremia: natural history and attributable mortality. Clin Infect Dis 1996;23:1234-9.

33. Lodise T.P., Mckinnon P.S., Tam V.H., Rybak M.J. Clinical outcomes for patients with bacteremia caused by vancomycin-resistant enterococcus in a level 1 trauma center. Clin Infect Dis 2002;34:922-9.

34. Carmeli Y., Eliopoulos G., Mozaffari E., Samore M. Health and economic outcomes of vancomycin-resistant enterococci. Arch Intern Med 2002;162:2223-8.

35. Schumacher G.E. Therapeutic drug monitoring. Connecticut: Appleton \& Lange; 1995.

36. Melo D.O., Sasaki M., Grinbaum R.S. Vancomycin use in a Hospital with High Prevalence of Methicillin-Resistant Staphylococcus aureus: Comparison with Hospital Infection Control Practices Advisory Committee Guidelines (HICPAC). Braz J Infect Dis 2007;11(1):53-7.

37. Evans M.E., Kortas K.J. Vancomycin use in a university medical center: comparison with Hospital Infection Control Practices Advisory Committee Guidelines. Infect Control Hosp Epidemiol 1996; 17 :356-9.

38. Rehm S.J. Two new treatment options for infections due to drugresistant Gram-positive cocci. Cleve Clin Med 2002; 69:397-413.

39. Singer M.V., Haft R., Barlan T., et al. Vancomycin control measures at a tertiary-care hospital: impact of interventions on volume and patterns of use. Infect Control Hosp Epidemiol 1998;19:248-53.

40. Montravers P., Gauzit R., Muller C., et al. Emergence of antibioticresistant bacteria in cases of peritonitis after intraabdominal surgery affects the efficacy of empirical antimicrobial therapy. Clin Infect Dis 1996;23:486-94.

41. Van Houten N.A., Uiterwaal C.S.P.M., Heesen G.J.M. et al. Does the empiric use of vancomycin in pediatrics increase the risk for Gram-negative bacteremia? Pediatr Infect Dis J 2001;20:171-7.

42. Watanakunakorn, C. Prescribing pattern of vancomycin in a community teaching hospital with low prevalence of vancomycin resistant enterococci. Infect Control Hosp Epidemiol 1997;18:767-9.

43. Fridkin S.K., Steward C.D., Edwards J.R. et al. Surveillance of antimicrobial use in United States hospitals: project ICARE phase 2. Clin Infect Dis 1999;29:245-52. 